\title{
A COMPARATIVE STUDY OF MIMO CAPACITY WITH DIFFERENT ANTENNA TOPOLOGIES
}

\author{
Liang Xiao, Lin Dai, Hairuo Zhuang, Shidong Zhou, Yan Yao \\ Tsinghua University, 100084, Beijing, CHINA, \\ Xiaoliang00@mails.tsinghua.edu.cn. pailin98@mails.tsinghua.edu.cn
}

\begin{abstract}
Multiple input/multiple output (MIMO) is a hot topic in wireless communication and has great advantage in capacity. We investigate the influence of antenna topology on MIMO capacity and by comparing three topology schemes we find that selecting a proper antenna topology increases the capacity evidently. Three schemes under study are the traditional point-to-point concentrated antenna topology (CON), the multipoint-to-point distributed antenna topology (DIS) and the clustered antenna topology (CLUSTER). We explore their average capacity under different antenna number and power allocation methods. Simulation results show that DIS and CLUSTER excel the traditional concentrated MIMO structure in capacity.
\end{abstract}

Keywords - MIMO capacity distributed antenna waterfilling equal-power allocation

\section{INTRODUCTION}

Multiple input/multiple output (MIMO) significantly increases the channel capacity and improves the reliability of the wireless link. The MIMO capacity in a narrow-band Rayleigh-fading channel was analyzed in [1][2] and that in other wireless channel models was investigated in [3][4][5].

However, most existing researches focus on the pointto-point MIMO systems and few aims at the influence of the antenna topology on the MIMO capacity. Therefore, in this paper we analyze the relationship between MIMO channel capacity and antenna topology based on the channel model considering path loss, lognormal shadowing and Rayleigh fading. Three antenna topology schemes are investigated: the concentrated topology $(\mathrm{CON})$, the distributed topology (DIS) and the clustered topology (CLUSTER). Both $\overline{C_{e}}$, average capacity with equal-power allocation and $\overline{C_{w}}$, average capacity with water-filling power allocation are considered.

To compare the capacity of three topology fairly, we suppose in our simulation that both the number of transmit antenna and the overall transmit power in unit area should be equal for three schemes. Simulation results show that $\overline{C_{w}}$ of DIS is greater than that of CLUSTER by about $2 \mathrm{bps} / \mathrm{Hz}$, while CLUSTER also has about $1.5 \mathrm{bps} / \mathrm{Hz}$ capacity gain over CON. As to $\overline{C_{e}}$, the results are similar. Through further analysis and simulation, we find that DIS, as well as CLUSTER, reduces average access distance of the receiver and thus decreases the required Tx power, which leads to the improvement in capacity. We also find that when the number of Tx antenna increases, the DIS capacity with equal power allocation decreases. That is because it distributes some transmit power to the Tx antennas that are not nearest to the receiver. Therefore, DIS should adopt the water-filling power allocation strategy in order to exert its capacity advantage.

In Section II, we give the channel model and review the channel capacity formulas with different power allocation schemes. In Section III we illustrate the topology and power control method of three schemes, as well as the assumptions made in the comparison. The simulation results are presented and discussed in Section IV. Finally, a brief summary of the paper is made in Section $\mathrm{V}$.

\section{CHANNEL MODEL AND CHANNEL CAPACITY}

The following notations are used throughout the paper: ' for vector transpose, * for transpose conjugate.

In this paper, we investigates the single-user (M, N) system with $\mathrm{M}$ transmit $(\mathrm{Tx})$ antennas and $\mathrm{N}$ receiver $(\mathrm{Rx})$ antennas. The channel is assumed to be linear and timeinvariant, described with discrete-time equivalent model:

$$
Y=H X+Z
$$

where, $X=\left[x_{1}, x_{2}, x_{3}, \ldots, x_{M}\right]^{\prime}$ is a $M \times 1$ vector and its $\mathrm{i}$-th component represents the signal from the ith Tx antenna. Y and $\mathrm{Z}$ are the $N \times 1$ vectors. Y presents the Rx signal and $\mathrm{Z}$ presents the additive white Gaussian noise (AWGN) with zero mean and unit variance.

Since our research is not limited to the point-to-point channel, we consider path-loss, Rayleigh fading and lognormal shadowing in the channel model. The channel is assumed to be quasi-static, namely, it is constant in a frame and varies from frame to frame. Particularly, the channel gain from the transmitter $\mathrm{i}$ to the receiver $\mathrm{j}$ can be expressed as

$$
h_{i j}=r_{i j}^{-\alpha / 2} \cdot \beta_{i j}
$$


where $r_{i j}$ is the distance from the $i$-th $\mathrm{Rx}$ antenna to the $j$ th Tx antenna. $\alpha$ is the path-loss exponent and in this paper it is set to 4. $\beta_{i j}$ is a complex random variable influenced by both the fast fading and log-normal shadowing. Its amplitude is Rayleigh distributed whose mean square value is lognormal random variable with zero mean and variance $\sigma_{s}[7]$.

Channel capacity is defined as the highest rate at which information can be sent with arbitrarily low probability of error [8]. It can be viewed as a random variable determined by the specific realization of channel matrix that changes with the location of the receiver, SNR, $(\mathrm{M}, \mathrm{N})$ and etc. In order to counteract the receiver location factor, we investigate a large volume of realizations and let the receiver distribute evenly in the area.

There are several strategies to distribute the transmit power over the antennas: if the transmitter has perfect knowledge about the channel, $C_{w}$ can be achieved by using the water-filling power allocation; otherwise, if the channel information is unknown to the receiver, the equal power allocation method should be adopted to attain the capacity $C_{e}$.

From [2][9], we can get the capacity with equal power allocation

$$
C_{e}=\log \operatorname{det}\left(I_{N}+\frac{P}{M \sigma^{2}} \mathbf{H H}^{*}\right)=\sum_{i=1}^{k} \log \left(1+\frac{P}{M \sigma^{2}} \lambda_{i}\right)
$$

, where $k$ is the rank of channel and $\lambda_{i}$ is the eigenvalue of HH$^{*}$.

[4] gives the capacity with water-filling power allocation

$$
C_{w}=\sum_{i=1}^{k}\left(\log \left(\mu \lambda_{i}\right)\right)
$$

where $\mu$ satisfies

$$
\sum_{i=1}^{k}\left(\mu-\lambda_{i}^{-1}\right)^{+}=\frac{P}{\sigma^{2}}
$$

and $\mathrm{a}^{+}$means $\max \{0, \mathrm{a}\}$.

\section{ANTENNA TOPOLOGY MODEL}

This paper investigates three antenna topology schemes, CON, DIS and CLUSTER. CON is the traditional point-topoint MIMO topology shown in Fig. 1 (a), with all the transmit antennas co-located as an antenna-set. The distance from one antenna-set to the neighbouring antenna-set is
$2 * \mathrm{R}_{1}$ and the distance from the antenna-set to the receiver is $r_{1}$. The convenient power control method is adopted to overcome path-loss: the overall transmit power from $\mathrm{M}$ antennas is proportional to $r_{1}^{4}$, that is

$$
P=P_{0 c} \cdot r_{1}^{4}
$$

The second topology DIS shown in fig.1 (b) was introduced to exploit the spatial resource. In the multipointto-point topology, all the antennas are distributed uniformly in the area and $\mathrm{M}$ antennas with best channel gains are selected to send the same signals to the receiver with different power [6]. The distance between two neighbouring antennas is $2 * R_{2}$. Because the distances from the receiver to the $M$ transmit antennas are not equal, the transmit power control scheme in DIS is different from the traditional pointto-point systems. We select from many power control options a method that utilizes the advantage of short average access distance in DIS. Suppose the distance from the receiver to the nearest transmit antenna is $r_{2}$, then the overall transmit power is $P=P_{0 D} \cdot r_{2}^{4}$. By this means, the path loss of the nearest transmit antenna is counteracted, while the capacity of DIS is still affected by other antennas' path-loss.

The third topology CLUSTER shown in Fig.1(c) is a trade-off between DIS and CON. Instead of only one antenna-set in CON, $T^{*}$ antennas-sets are selected with $M^{*}$ $\left(M^{*}>1, T^{*} \times M^{*}=M\right)$ antennas in each set. The definition of $r_{3}$ is similar to $r_{2}$ in DIS and we let $P=P_{0 U} \cdot r_{3}^{4}$.

In order to make a fair comparison of the topology schemes, we suppose that both the number of transmit antenna and the total transmit power in unit area are equal. That is,

$$
\frac{M}{R_{1}^{2}}=\frac{1}{R_{2}^{2}}=\frac{M^{*}}{R_{3}^{2}}
$$

$$
\begin{aligned}
& \frac{\left(\int_{0}^{2 \pi} \int_{0}^{R_{1}} P_{0 \mathrm{C}} \times \mathrm{r}^{4} \times \mathrm{r} d \mathrm{r} d \theta\right)}{R_{1}^{2}}=\frac{\left(\int_{0}^{2 \pi} \int_{0}^{R_{2}} P_{0 \mathrm{D}} \times \mathrm{r}^{4} \times \mathrm{r} d \mathrm{r} d \theta\right)}{R_{2}^{2}} \\
& =\frac{\left(\int_{0}^{2 \pi} \int_{0}^{R_{3}} P_{0 \mathrm{U}} \times \mathrm{r}^{4} \times \mathrm{r} d \mathrm{r} d \theta\right)}{R_{3}^{2}}
\end{aligned}
$$

Then combining with (7)(8), we can get the relationship among $R_{1}, R_{2}$ and $R_{3}$ as well as the relationship among the overall transmit power of the three schemes:

$$
P_{0 \mathrm{C}} \cdot M^{3}=M \cdot P_{0 \mathrm{D}}=P_{0 \mathrm{U}} \cdot M^{* 4}
$$




\section{SIMULATION RESULTS}

In this section, we present the simulation results of the channel capacity in the proposed antenna topology schemes. In the simulation, we set $\sigma^{2}=1, \sigma_{s}=8 \mathrm{~dB}, M^{*}=2$ and $R_{2}=1$. Then by equation (7), we have $R_{1}=2$ and $R_{3}=\sqrt{2}$ for the case $\mathrm{M}=4$. The $\mathrm{SNR}$ ranges from $5 \mathrm{~dB}$ to $15 \mathrm{~dB}$.

Firstly, we make a capacity comparison for three antenna topologies in figure 2 , where $\overline{C_{w}}$ and $\overline{C_{e}}$ are denoted as $\mathrm{C}$ and I, respectively. From the equation (9), we have $P_{0 \mathrm{C}}=P_{0 \mathrm{D}} / 16$ and $P_{0 \mathrm{U}}=P_{0 \mathrm{D}} / 4$. Let $S N R=P_{0 \mathrm{D}} / \sigma^{2}$. From $\sigma^{2}=1$ we have $S N R=P_{0 \mathrm{D}}$. This figure illustrates that DIS and CLUSTER are superior to CON. $\overline{C_{w}}$ and $\overline{C_{e}}$ of DIS are about $3.5 \mathrm{bps} / \mathrm{Hz}$ and $1.5 \mathrm{bps} / \mathrm{Hz}$ greater than CON. The corresponding values of CLUSTER gaining over $\mathrm{CON}$ are $1.5 \mathrm{bps} / \mathrm{Hz}$ and $1 \mathrm{bps} / \mathrm{Hz}$, respectively. This capacity benefit of DIS as well as CLUSTER is due to its topology characters: this distributed antenna topology reduces average access distance of the receiver and thus the required transmit power, which improves the channel capacity.

Afterwards, we choose four typical (M, N) systems: (2, $2),(2,4),(4,2)$ and $(4,4)$ to further explore the capacity of CON and DIS.

Fig. 3 shows how the CON capacity with water-filling power allocation increases with the number of either transmit or receive antennas. We can see that the CON capacity of $(2,4)$ system with water-filling equals to the capacity of $(4,2)$. Therefore, in this point-to-point symmetrical structure, the capacity improvement from the antenna diversity of the transmit side equals to that from the diversity of the receiver side.

Fig.4 presents the CON capacity with equal power allocation, where $\overline{C_{e}}$ of $(2,4)$ system gains over $(4,2)$ system for about $1 \mathrm{bps} / \mathrm{Hz}$. This figure shows that with the equal-power allocation, the capacity increases less by rising the number of transmit antennas than that by rising the number of the receive antennas.

Fig.5 and Fig.6 discuss the DIS capacity with waterfilling power allocation and equal power allocation respectively. We can see that the DIS capacity value in these figures is smaller than the corresponding CON capacity value in Fig. 3 and Fig.4, contrary to what we saw in Fig. 2. For example, when $\mathrm{M}=2, \mathrm{~N}=2$ and $\mathrm{SNR}=5 \mathrm{~dB}, \overline{C_{e}}$ of DIS in fig.6 is $1.5 \mathrm{bps} / \mathrm{Hz}$ less than that of $\mathrm{CON}$ in Fig.4. This discrepancy lies in the power control scheme adopted by these figures that is unfair to compare the capacity of DIS with that of CON. In these figures, the fairness rule of equation (9) is not observed and we just let $P_{0 \mathrm{C}}=P_{0 \mathrm{D}}$. Therefore, more overall transmit power in the same area is allocated to CON than to DIS.
Fig.5 shows that the DIS capacity with water-filling increases slightly with $\mathrm{M}$, e.g. $\overline{C_{w}}$ of the $(2,4)$ system is approximate to that of $(4,4)$. The reason lies in the different access distances and path-loss of the transmit antennas. If $\mathrm{M}$ increases, so does the number of Tx antennas with greater path-loss, while the antenna with the least path-loss doesn't change. Since the channel capacity with water-filling power allocation is mainly decided by the latter, it cannot obtain much gain from the rise of $\mathrm{M}$.

From figure 6, we can see $\overline{C_{e}}$ of $(2,2)$ is about $1 \mathrm{bps} / \mathrm{Hz}$ greater than $(4,2)$ and $\overline{C_{e}}$ of $(2,4)$ is greater than $(4,4)$ when SNR is not very large. It is contrary to the common belief that transmit diversity enhances capacity. With the equal power allocation instead of the optimized water-filling allocation, part of the transmit power in DIS is distributed to the transmit antennas with greater path-loss. Therefore, the capacity with equal-power allocation decreases with the number of Tx antennas, when the SNR is not large enough to ignore the effects of path-loss.

\section{CONCLUSIONS}

We studied the average capacity of three MIMO antenna topology schemes: DIS, CON and CLUSTER. It was found that if the total transmit power as well as the number of transmit antenna in unit area is equal for three topologies, DIS and CLUSTER with adequate power control improves the MIMO capacity evidently. That is because compared with $\mathrm{CON}$, they reduce the access distance for the receiver and thus reduce the required transmit power. The topology of CLUSTER is a trade-off between CON and DIS, and so does its performance in capacity.

We also found that the capacity benefit of the transmit antenna diversity almost equals to that of the receive antenna diversity in CON. Nevertheless, in DIS using the water-filling power allocation, the former is far less than the latter. Moreover, the DIS capacity with equal-power allocation significantly decreases with the number of Tx antennas when SNR is not large enough to ignore the effects of path-loss.

\section{REFERENCES}

[1] I. E. Telatar, "Capacity of multi-antenna Gaussian channels", AT\&T-BELL Lab Internal Tech Memo, June 1995.

[2] G. J. FOSCHINI, M. J. GANS, "On Limits of Wireless Communications in a Fading Environment when Using 
Multiple Antennas", Wireless Personal Communications, vol.6, no.3, pages 311-335, March 1998.

[3] Chen-Nee Chuah and David N.C.Tse, Capacity Scaling in MIMO Wireless Systems Under Correlated Fading, IEEE Transactions On Information Theory, vol.48, NO.3, March 2002.

[4] C. Chuah, J. M. Kahn and D. Tse, "Capacity of multiantenna array systems in indoor wireless environment", in Proc. Globecom'98, Sydney, Australia, pp. 1894 -1899, Nov., 1998.

[5] R. S.Blum, "MIMO Capacity with Interference", in Proc. CISS'02, Princeton, NJ, March 2002.

[6] L. Dai, S. Zhou, and Y. Yao, "Capacity with MRC-based Macrodiversity in CDMA Distributed Antenna Systems ”, to be published, Globecom'02, Taipei, Nov. 2002.

[7] L. Dai, H. Zhuang, S. Zhou, and Y. Yao, "Reverse-link Capacity with MRC-based Macrodiversity in DS-CDMA Cellular Systems", in Proc. VTC'02-spring, Birmingham, AL., pp. 255-259, May 2002.

[8] T.M.Cover and J.A.Thomas, Elementary of Information Theory, John Wiley \& Sons, New York, 1991.

[9] D. A. Gore, R. U. Nabar and A. Paulraj, "selecting an optimal set of transmit antennas for a low rank matrix channel", in Proc. ICASSP'00, Istanbul, Turkey, pp.27852788, June 2000.

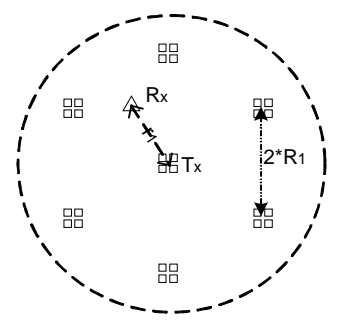

(a) $\mathrm{CON}$

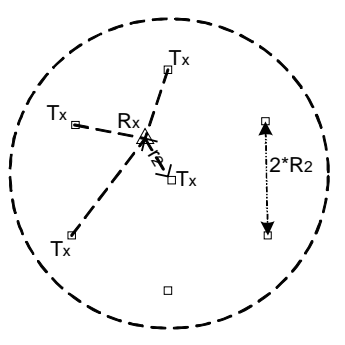

(b) DIS

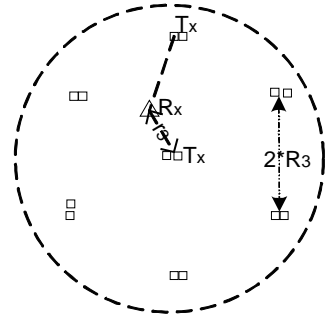

(c) CLUSTER

Fig 1. MIMO antenna topology

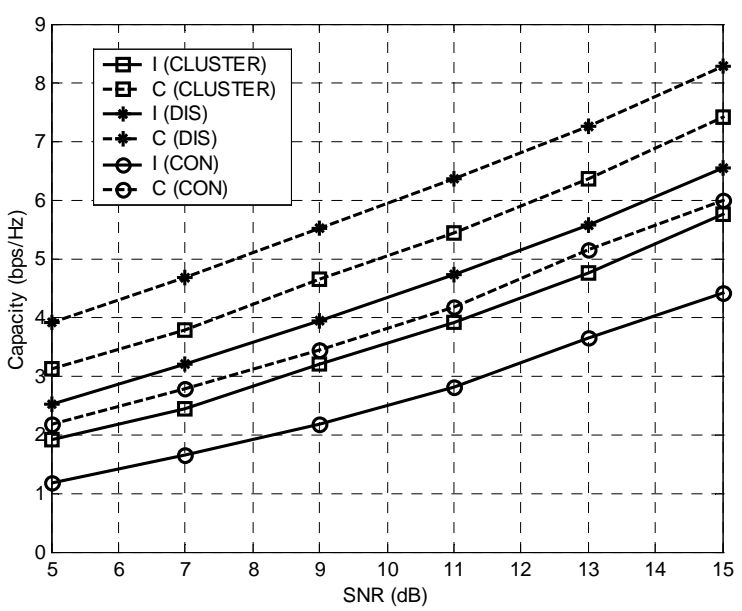

Fig. 2. $\overline{C_{w}}$ (I) and $\overline{C_{e}}(\mathrm{C})$ in DIS, CON and CLUSTER vs. $\mathrm{SNR}, \mathrm{M}=4, \mathrm{~N}=2$

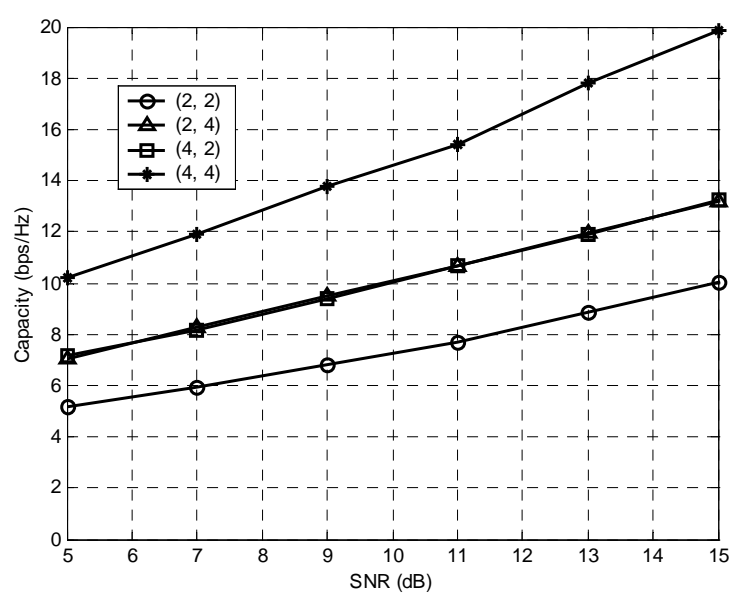

Fig. 3. $\overline{C_{w}}$ vs. SNR in CON 


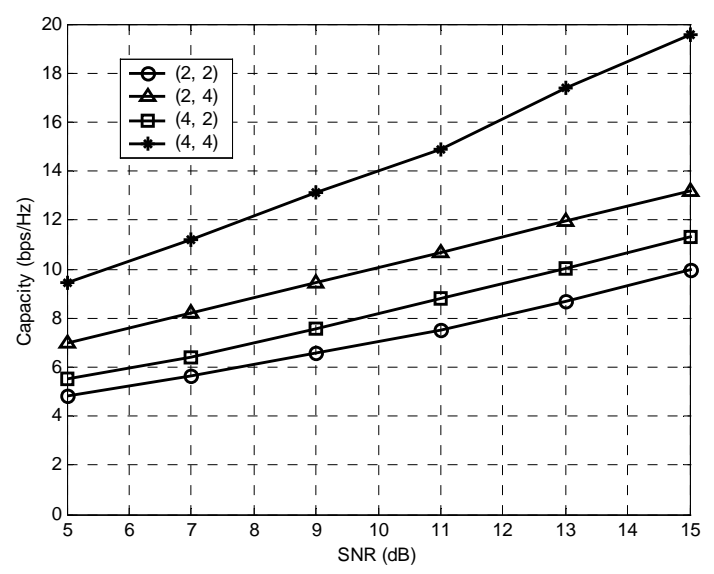

Fig. 4. $\overline{C_{e}}$ vs. SNR in CON

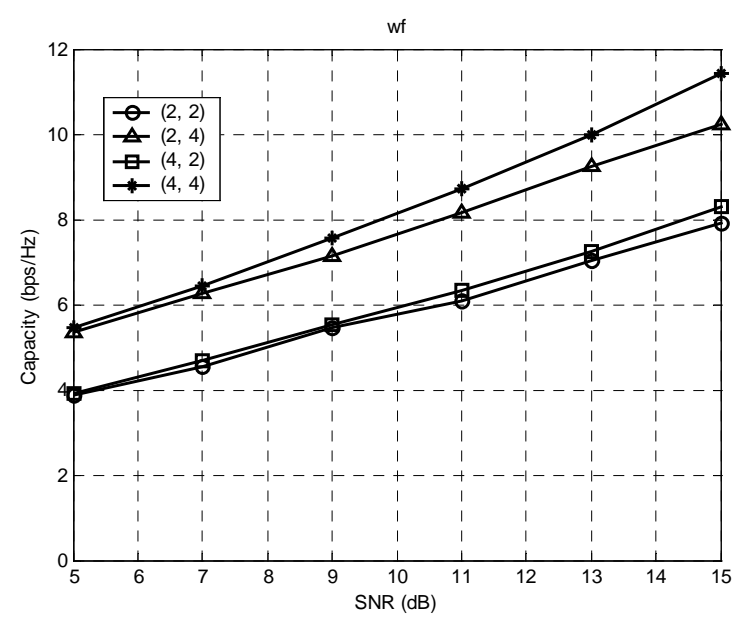

Fig. 5. $\overline{C_{w}}$ vs. SNR in DIS.

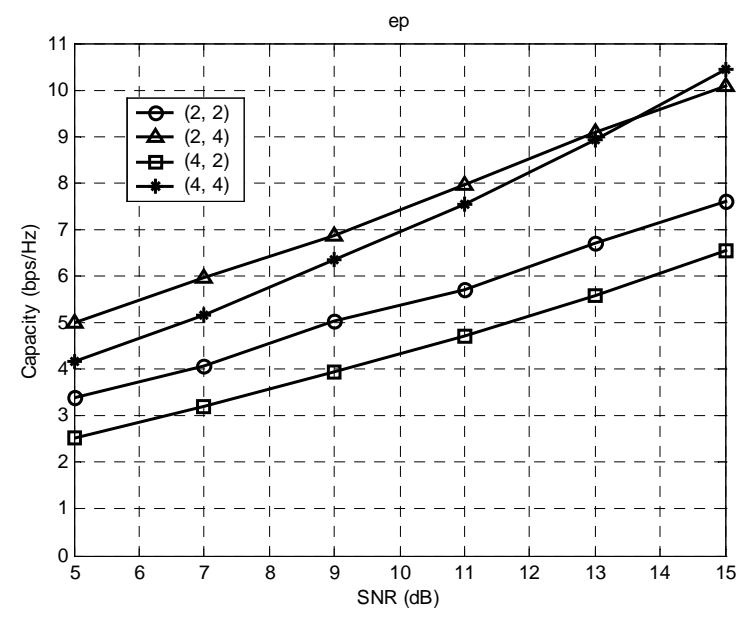

Fig. 6. $\overline{C_{e}}$ vs. SNR in DIS 